\title{
Forthcoming Lab on a Chip tutorial series on acoustofluidics: Acoustofluidics-exploiting ultrasonic standing wave forces and acoustic streaming in microfluidic systems for cell and particle manipulation
}

Bruus, Henrik; Dual, Jurg; Hawkes, Jeremy; Hill, Martyn; Laurell, Thomas; Nilsson, Johan; Radel, Stefan; Sadhal, Satwindar; Wiklund, Martin

\section{Published in:}

Lab on a Chip

Link to article, DOI:

$10.1039 / \mathrm{c} 1 \mathrm{lc} 90058 \mathrm{~g}$

Publication date:

2011

Document Version

Publisher's PDF, also known as Version of record

Link back to DTU Orbit

Citation (APA):

Bruus, H., Dual, J., Hawkes, J., Hill, M., Laurell, T., Nilsson, J., Radel, S., Sadhal, S., \& Wiklund, M. (2011). Forthcoming Lab on a Chip tutorial series on acoustofluidics: Acoustofluidics-exploiting ultrasonic standing wave forces and acoustic streaming in microfluidic systems for cell and particle manipulation. Lab on a Chip, 11(21), 3579-3580. https://doi.org/10.1039/c1lc90058g

\section{General rights}

Copyright and moral rights for the publications made accessible in the public portal are retained by the authors and/or other copyright owners and it is a condition of accessing publications that users recognise and abide by the legal requirements associated with these rights.

- Users may download and print one copy of any publication from the public portal for the purpose of private study or research.

- You may not further distribute the material or use it for any profit-making activity or commercial gain

- You may freely distribute the URL identifying the publication in the public portal 


\title{
Forthcoming Lab on a Chip tutorial series on acoustofluidics: Acoustofluidics-exploiting ultrasonic standing wave forces and acoustic streaming in microfluidic systems for cell and particle manipulation
}

\author{
Henrik Bruus, ${ }^{a}$ Jurg Dual, ${ }^{b}$ Jeremy Hawkes, ${ }^{c}$ Martyn Hill, ${ }^{d}$ Thomas Laurell, ${ }^{e}$ Johan Nilsson, ${ }^{e}$ Stefan Radel, ${ }^{f}$ \\ Satwindar Sadhal ${ }^{g}$ and Martin Wiklund ${ }^{h}$
}

DOI: $10.1039 / \mathrm{c} 1 \mathrm{lc} 90058 \mathrm{~g}$

This Editorial is the forerunner for a series of tutorial papers on fluidics combined with ultrasonic standing wave technology, termed acoustofluidics. The goal of the series is to review the state of art and to provide a point of entry for newcomers, be it students or senior researchers, into this rapidly developing research field. The initiative to do this originated in June 2010 as the authors convened for the first time for a week at CISM (International Centre for Mechanical Sciences) in northern Italy, giving a lecture series on the theory and

${ }^{a}$ Dept. Micro- and Nanotechnology, Technical University of Denmark, DTU Nanotech Building 345 B, DK-2800, Kongens Lyngby, Denmark

${ }^{b}$ Institute of Mechanical Systems, Dept. of Mechanical and Process Engineering, ETH

Zurich, Tannenstr. 3, 8092, Zurich, Switzerland

${ }^{c}$ Manchester Interdisciplinary Biocentre, The

University of Manchester, 131 Princess Street,

Manchester, M1 7DN, UK

${ }^{d}$ Engineering Sciences, University of

Southampton, Southampton, SO17 1BJ, UK

${ }^{e}$ Dept. Measurement Technology and Industrial

Electrical Engineering, Div.

Nanobiotechnology, Lund University,

${ }^{f}$ Institute of Chemical Technologies and

Analytics \& Institute of Applied Physics, Vienna University of Technology, Austria

${ }^{g}$ Dept. of Aerospace \& Mechanical Engineering, University of Southern California, Los Angeles, California, 90089-1453, USA

${ }^{h}$ Dept. of Applied Physics, Royal Institute of

Technology, KTH-Albanova, SE-106 91,

Stockholm, Sweden applications of ultrasonic standing wave technology and microfluidics for students active in the field. The impression of the total material presented on this single occasion was overwhelming and therefore we decided to communicate the essence of our lectures in a comprehensive and digestible format to the microfluidics and lab-on-a-chip community, an initiative that has been positively supported by the RSC Lab on a Chip journal.

Lab-on-a-chip technology and microfluidics developed rapidly in the 1990s, and the turn of the millennium saw an expansion of the field from analytical chemistry to target challenges within bioanalysis, cell biology, and clinical applications. With this development, new requirements emerged for the manipulation and handling of particles and cells in microfluidic systems. Today hydrodynamic, electric, dielectric, and magnetic actions are all used. Acoustic techniques have also entered this arena in the form of acoustophoresis or acoustic trapping along with the exploitation of phenomena such as acoustic streaming and cavitation. In this tutorial series it is the ultrasonic standing wave generated forces and acoustic streaming that are considered in detail.

Ultrasonic standing waves have proven to be a simple and robust strategy to accomplish chip integrated separation, focusing, concentration, removal, trapping and/or aggregation of particulate matter, for example biological cells or functionalized microbeads. In addition, through the generation of acoustic streaming they provide a means of fluid movement and mixing. The frequencies used are generally above $1 \mathrm{MHz}$ and therefore most often beyond the range of cavitation and its adverse effects on cell viability.

The ability to manipulate particles and fluids by means of ultrasonic standing wave forces is well known and has been explored by many eminent scientists including Chladni, Savart, Faraday, Kundt, Rayleigh, King and Gorkov. The theory of ultrasonic standing wave forces and the action on micro particles dates back to the mid-1930s, with significant additions in the 1950s and $60 \mathrm{~s}$ to the radiation force theory of elastic particles in viscous liquids. An important step was taken by Coakley as the exploitation of ultrasonic standing wave forces in small fluidic devices were approached at the turn of the millennium.

In the last decade, developments in computer power and software have led to an increasing number of numerical simulations of acoustic fields in microsystems and acoustophoresis of particles within these fields. These are now at a level where in silico predictions of ultrasonic particle manipulation in microfluidic systems agree reasonably well with experimental observations 


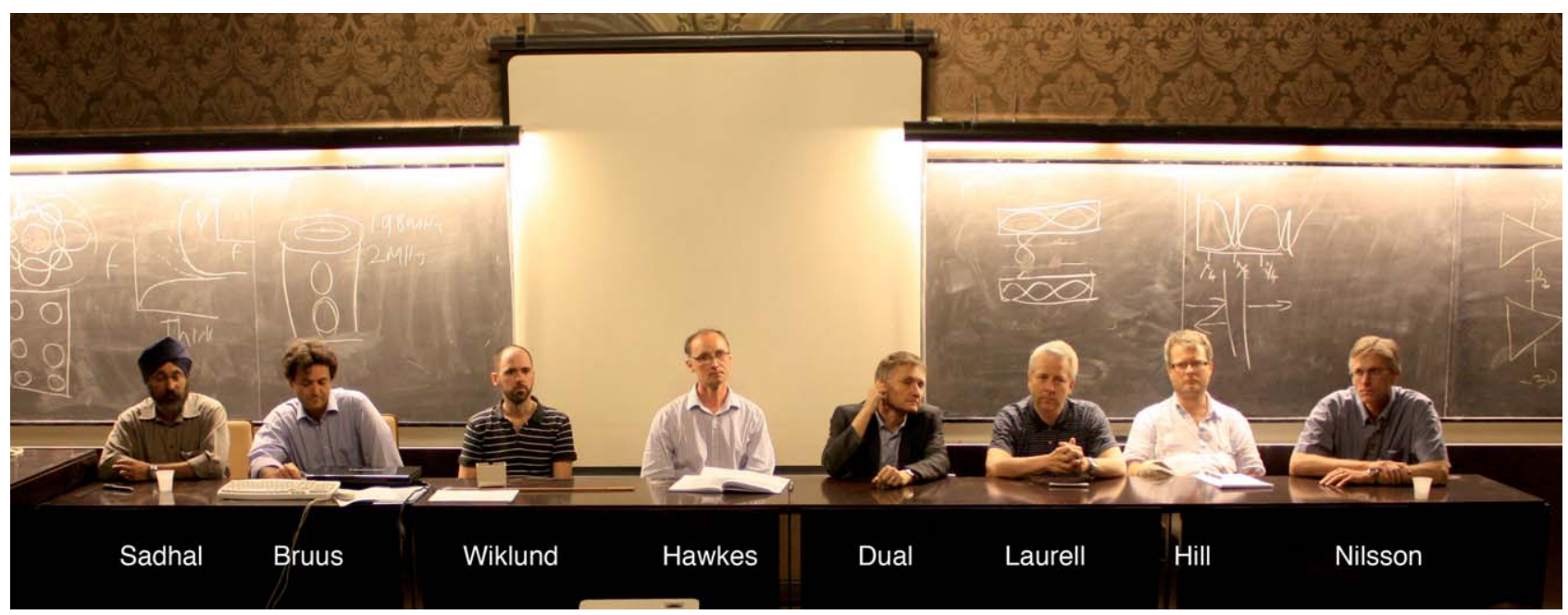

The authors on the podium at the International Centre for Mechanical Sciences (CISM) in Udine, Italy, June 2010. Photo credit: Carl Grenvall.

and thus begin to offer a powerful tool for acoustofluidic system design.

In this tutorial series we will describe both the underlying theory of ultrasonic standing wave physics at its current stage of development and how this can be applied in microfluidic systems to accomplish elaborate particle handling functions. Fundamental fluid mechanics theory and elasticity theory will be conveyed from a microfluidic perspective. Our goal is to balance theory with relevant experimental work to give our readers a comprehensive appreciation of the technology. Various means to capitalise on the primary acoustic radiation force will be considered such as continuous flow half wavelength focusing, single node acoustic trapping, and multinode sedimentation and particle guiding systems with emphasis on cell handling. With this primary radiation force, a wide range of bioanalytical detection strategies can be achieved in combination with techniques such as bead-based biomarker assaying, obtaining high sensitivity detection, and decomplexing of biological samples prior to analytical readout. The possibilities and proposed approaches for harnessing secondary (inter-particle) radiation forces will also be illuminated.

Beside the ultrasonic standing wave, the occurrence of acoustic streaming is of importance with respect to forces exerted on particles within acoustic resonators. Moreover, the exploitation of streaming offers opportunities to control convection of the microenvironments. We will therefore give an extensive outline of the underlying physics of acoustic streaming and also look into some basic applications that successfully use streaming. Acoustic streaming and secondary radiation forces can be deliberately used to enhance performance, but their influence can often appear unexpectedly and confuse the unwary investigator. Hence we need to know when these phenomena can play a role in our systems and avoid the potential pitfalls.
The tutorial series will also give direct advice on the design and fabrication of experimental set-ups allowing the reader to appreciate critical design parameters of acoustic resonators and their coupling to the ultrasonic transducers. Various methods for the experimental characterization of resonator systems will be described and advice will be given on important considerations in the design of the optical imaging systems that complement the acoustofluidic set-ups.

In conclusion, we hope that this tutorial series will serve as a base for a wider understanding of the area of acoustofluidics and the opportunities that the combination of ultrasonic standing wave technology, microfluidics and microtechnology offer to the life sciences and analytics.

We hope Lab on a Chip readers will enjoy this tutorial series on acoustofluidics and that they will find it informative and enabling for their research activities. 